\title{
ANALYTICAL METHOD DEVELOPMENT BY SOLID PHASE EXTRACTION AND HIGH- PERFORMANCE LIQUID CHROMATOGRAPHY FOR DIPHENHYDRAMINE QUANTIFICATION IN SYRUPS
}

\author{
MARCELA HURTADO*, JOSE RAUL MEDINA
}

Departamento de Sistemas Biológicos, Universidad Autónoma Metropolitana-Xochimilco, Mexico City, Mexico Email: mhurtado@correo.xoc.uam.mx

Received: 31 Aug 2017, Revised and Accepted: 10 Oct 2017

\begin{abstract}
Objective: To develop a method for the determination of diphenhydramine hydrochloride in syrups.

Methods: The developed method is based on the active ingredient recovery from the pharmaceutical formulation using a cartridge for selective solid phase extraction. This procedure is simple and fast as compared to the methods that require liquid/liquid extraction. Once the sample is eluted from the cartridge, it is injected directly into a reversed-phase chromatographic system containing a mixture of acetonitrile: 10 mmol ammonium acetate: triethylamine 60:39.5:0.5.
\end{abstract}

Results: The proposed method fulfilled the validation parameters (linearity $\mathrm{R}^{2}>0.99$, precision CV $<2 \%$ and accuracy $98-102 \%$ )

Conclusion: The proposed method proved to be reliable when it was applied in the quantification of diphenhydramine hydrochloride of two commercial syrup products.

Keywords: Diphenhydramine, Pre-treatment, Solid phase extraction, Syrup

(C) 2017 The Authors. Published by Innovare Academic Sciences Pvt Ltd. This is an open access article under the CC BY license (http://creativecommons.org/licenses/by/4.0/) DOI: http://dx.doi.org/10.22159/ijap.2017v9i6.20577

\section{INTRODUCTION}

Diphenhydramine is an antihistamine that is most useful in the treatment of acute types of allergies and symptoms of rhinitis, itching and conjunctivitis. It has antitussive activity and relieves a cough caused by bronchial and laryngeal irritation, common cold or inhaled agents [1]. It is also used to prevent and treat kinetosis and vertigo and is usually included in commercial products against insomnia for its sedative action [1].

One of the pharmaceutical dosage forms in which this active ingredient is administered is syrup $(0.25 \mathrm{~g} / 100 \mathrm{ml})$. The children dosage is $1.25 \mathrm{mg} / \mathrm{kg}$ of weight, 4 times a day, whereas the adult dose varies from 25 to $50 \mathrm{mg}, 3$ or 4 times a day [2].

There are various methods of analysing different pharmaceutical presentations of diphenhydramine hydrochloride by using highperformance liquid chromatography (HPLC) and capillary electrophoresis (CE). In the particular case of syrups, the methods often consider just one step of syrup dilution [3-5], which happens to be extremely simple and does not require any pre-treatment of the sample. However, diphenhydramine has the molar extinction coefficient of about $500 \mathrm{l} / \mathrm{mol} \mathrm{cm}\left(A_{1}^{1 \%}=17\right)$ [6] at $260 \mathrm{~nm}$, which means that, in order to have significant signals at such wavelength, relatively high concentrations of the active ingredient are required. According to the Beer's law, the response of a sample in absorbance units is proportional to the concentration, the optical path length and the extinction coefficient. Therefore, smaller values of extinction coefficients mean that a higher concentration of analyte will be necessary for a better response at the same optical path length. If the analyte concentration is lower than the one required to satisfy an adequate response at a certain wavelength, a wavelength with an extinction coefficient that is more favourable for determination should be selected in accordance with the concentration of the final sample dilution. With the methods reported previously [3-5], the wavelengths between 210 and $215 \mathrm{~nm}$ were lower selective because very simple chromophores are detected at such wavelengths.

The direct method could present variability problems or possible interferences in the analytical procedure, depending on the formulation. Therefore, samples should be submitted to a pretreatment so that clean samples can be achieved and analytical equipment maintained in better conditions, attaining better concentrations of the active ingredient and smaller interferences in the analysis. The method of analysis of diphenhydramine hydrochloride in syrups that is reported in Mexican Pharmacopeia $11^{\text {th }}$ Ed., uses liquid-liquid extractions and subsequently, a spectrophotometric analysis at $260 \mathrm{~nm}$ [7]. The liquid-liquid extraction is a separation method particularly used in liquid formulations that has some disadvantages, such as use of toxic solvents, need for more than one extraction and the subsequent necessity to either evaporate the solvent or, by changing the $\mathrm{pH}$, transfer the analyte to the aqueous phase for its measurement [7]. Some methods for diphenhydramine analysis propose the extraction of a coloured complex formed between the analyte and some dye, such as eriochrome black $\mathrm{T}$, bromocresol purple, methyl orange, bromocresol green and rose bengal, using organic solvents. These methods provide an improved capacity of detection and drug selectivity, but their extraction requires the use of an organic solvent of the formed complex [8-11].

The solid phase extraction (SPE) has been applied to concentrate different types of samples (biological fluids, contaminated waters, food, etc [12]), but it has also been very useful in removing compounds that interfere in complex matrices [13]. Syrups are very complex formulations that usually contain a wide spectrum of excipients; a pre-treatment of the formulation by SPE allows obtaining samples relatively free of interferences for their further analysis by HPLC. Given the advantages of SPE, such as: one-step recovery of almost $100 \%$ of the analyte, a small amount of solvent used and a great variety of sorbents that allow selective extraction of compounds, this work proposes a method for determination of diphenhydramine hydrochloride in syrups by SPE and HPLC. The method was validated in accordance with international criteria [14] for drug determination in two commercial syrups.

\section{MATERIALS AND METHODS}

\section{Reagents}

Diphenhydramine hydrochloride and lidocaine hydrochloride were purchased from Sigma Chemical Company. Triethylamine and ammonium acetate, reagent grade, were acquired from Merck, Mexico. Acetonitrile and methanol solvents, chromatographic grade, 
were from J. T. Baker, Mexico and the water utilized in all the cases was a chromatographic grade, obtained by using a Simplicity System equipment (Millipore Corporation, USA).

\section{Chromatographic conditions}

A Knauer chromatography system, equipped with a Lab Alliance series III isocratic pump was employed together with the variablewavelength Spectra-Physics SP450 UV/Vis Detector and Knauer D14163 Berlin Num. 78098 Manual Injector. Chromabond HR-XCW 3 $\mathrm{ml} / 200 \mathrm{mg}$ Macherey-Nagel was used for the extraction of 12-port Supelco SPE equipment. Chromatographic separation was performed in Agilent Zorbax Eclipse plus $\mathrm{C}_{18}$ column, $150 \times 4.6 \mathrm{~mm}$ with $3.5 \mu \mathrm{m}$ particle size. A mixture of acetonitrile: $10 \mathrm{mmol}$ ammonium acetate: triethylamine 60:39.5:0.5 v/v/v was employed as mobile phase [15]. The separation was performed at a flow rate of $1 \mathrm{ml} / \mathrm{min}$ and the detection, at $260 \mathrm{~nm}$.

\section{Preparation of sample}

Samples of two brands of diphenhydramine hydrochloride syrups were submitted to SPE using lidocaine hydrochloride aqueous solution as internal standard $(0.6 \mathrm{mg} / \mathrm{ml})$. Syrup volumes equivalent to $7.5 \mathrm{mg}$ of drug were diluted with $3 \mathrm{ml}$ of aqueous solution of the internal standard. Prior to drug extraction from the syrup sample, the cartridge with $3 \mathrm{ml}$ of acetonitrile and $7 \mathrm{ml}$ of distilled water was prepared and the sample mixed with an internal standard solution was immediately transferred to perform the following washes: $5 \mathrm{ml}$ of distilled water and $7 \mathrm{ml}$ of acetonitrile: water 50:50. The cartridge was vacuum dried and the sample eluted with $4 \mathrm{ml}$ of ammonia at $5 \%$ in acetonitrile. The sample was then received in a $5 \mathrm{ml}$ flask and diluted with the mobile phase. Finally, $20 \mu \mathrm{l}$ were injected into the chromatography system.

\section{Method validation}

Calibration curves were prepared in a concentration interval from 0.5 to $1.5 \mathrm{mg} / \mathrm{ml}$ with $0.3 \mathrm{mg} / \mathrm{ml}$ of lidocaine hydrochloride as an internal standard. The chromatography system characteristics were evaluated using the calibration curves, prepared in five replicates, of diphenhydramine hydrochloride with the internal standard in the working interval, whereas the standard solutions were diluted with a mixture of eluent and mobile phase equivalent to the final solvent of the samples (4:1 eluent: mobile phase). The regression values were determined together with the coefficients of variation (CV) of the replicates.

The precision of the analytical method was assessed in two different products, which were analysed for two days in six replicates, by calculating the percentage of recovery of the active ingredient with respect to the product labels and their $\mathrm{CV}$.

Method linearity was performed by measuring different volumes of syrup, equivalent to $40,60,100,111$ and $133 \%$ of the amount recommended in the method and by treating these samples in accordance with the methodology. The linearity was determined by calculating the coefficient of determination $\left(\mathrm{R}^{2}\right)$ between the amount of active ingredient added and the amount found in two different products. In order to determine the accuracy of the method, the recovery was calculated at three concentrations $(40,100$ and $133 \%)$ in three replicates, calculating in all cases the percentage of recovery and the $95 \%$ confidence interval. The method specificity was determined using samples of both commercial products and standard solutions, submitted to the same procedure and comparing the spectral analyses of the peaks. For the evaluation of the latter parameter, Varian Prostar equipment with 330 diode array detector was used.

\section{RESULTS}

Fig. 1 shows the chromatogram of the proposed method. The retention time for lidocaine hydrochloride was $4.45 \mathrm{~min}$ and for diphenhydramine hydrochloride, $6.06 \mathrm{~min}$ (relative retention time 1.36). The sample was added dextromethorphan hydrobromide because this drug is frequently added to diphenhydramine syrups. The retention time was 7.23 min (relative retention time 1.62). The resolution between the three compounds was above 2 and the peaks displayed adequate asymmetry factor.

\section{Method validation}

Fig. 2 exhibits the results of chromatography system linearity. The $\mathrm{R}^{2}$ of linear regression was 0.99 and the CV of the response factor was below $2 \%$. The $\mathrm{CV}$ was below $2 \%$ at all concentration levels examined. The chromatography system met the validation criteria for linearity and precision of the analytic system.

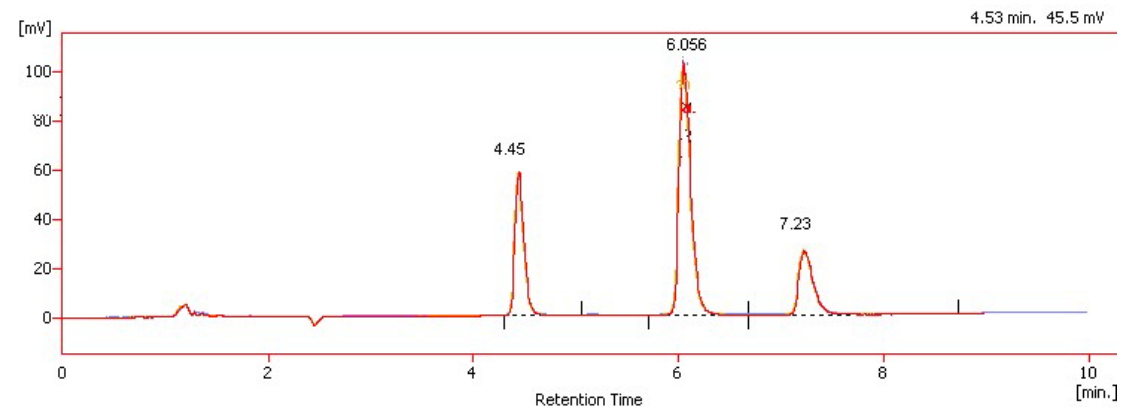

Fig. 1: Typical chromatogram, lidocaine hydrochloride $4.45 \mathrm{~min}$, diphenhydramine $6.05 \mathrm{~min}$, dextromethorphan $7.23 \mathrm{~min}$

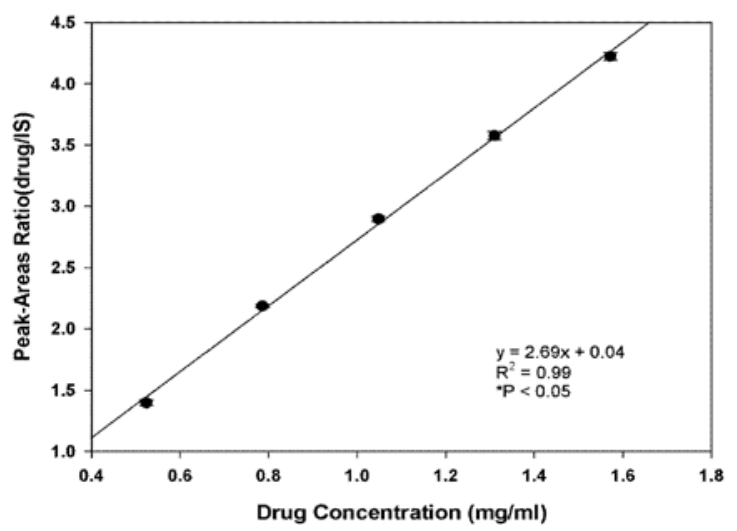

Fig. 2: Chromatographic system linearity. mean $\pm S D, n=5$ 
Table 1 shows the $\mathrm{CV}$ obtained for the percentage of recovery in one day, which was below $2 \%$. The variation during different days was also below $2 \%$. The precision of the method met the criteria of the precision and intermediate precision for both products.

The equation of the straight line obtained for the ratio of the amount added against the amount found was " $y=1.02 x+1.57$ with a $\mathrm{R}^{2}$ of 0.99 . The statistical analysis of the regression shows a slope non-different from 1 and an intercept non-different from zero (fig. 3).

Table 2 presents the results for accuracy, with a percentage of average recovery for the three levels of concentration tested of $99.97 \%$ and a confidence interval of 98.63 at $101.31 \%$. Thus, the method is considered to be within the parameters of accuracy.

The spectral analysis of syrup samples showed correspondence when compared to spectral analysis of standard samples.

Table 1: Precision and intermediate precision of the method

\begin{tabular}{|c|c|c|c|c|c|c|c|c|c|}
\hline & Day 1 & & & & Day 2 & & & & \\
\hline Product & Recovery & Average (\%) & SD & CV (\%) & Recovery & Average (\%) & SD & CV (\%) & Total CV (\%) \\
\hline \multirow[t]{5}{*}{ Benadryl } & 98.84 & 98.77 & 1.18 & 1.20 & 99.06 & 100.71 & 2.15 & 2.13 & 1.93 \\
\hline & 96.98 & & & & 103.52 & & & & \\
\hline & 100.31 & & & & 101.52 & & & & \\
\hline & 98.87 & & & & 101.35 & & & & \\
\hline & 98.85 & & & & 98.11 & & & & \\
\hline \multirow[t]{5}{*}{ Generic } & 98.10 & 99.66 & 1.78 & 1.78 & 97.03 & 100.15 & 2.00 & 2.00 & 1.8 \\
\hline & 99.20 & & & & 100.73 & & & & \\
\hline & 100.72 & & & & 102.05 & & & & \\
\hline & 102.23 & & & & 99.45 & & & & \\
\hline & 98.20 & & & & 101.49 & & & & \\
\hline
\end{tabular}

SD: standard deviation $(n=6), C V$ : coefficient of variation $(n=6)$

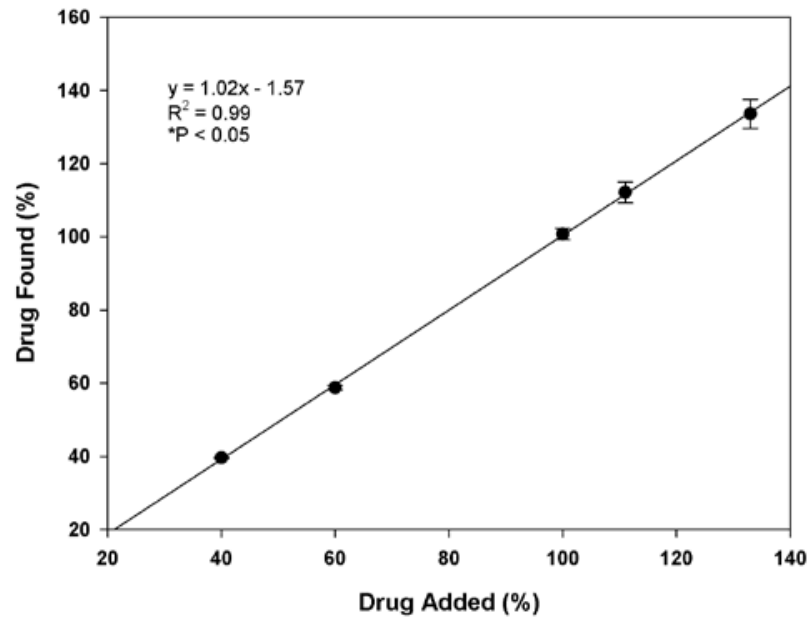

Fig. 3: Linearity of the proposed method. mean $\pm S D, n=3$

Table 2: Method accuracy

\begin{tabular}{lll}
\hline \% added & \% recovered & \% of recovery \\
\hline 40 & 39.60 & 99.00 \\
40 & 39.33 & 98.33 \\
40 & 39.80 & 99.50 \\
100 & 99.20 & 99.20 \\
100 & 100.67 & 100.67 \\
100 & 102.27 & 102.27 \\
133 & 136.00 & 102.26 \\
133 & 129.33 & 97.24 \\
133 & 134.67 & 101.25 \\
$\mathrm{CI}_{95 \%} 98.63-101.31(\mathrm{n}=9)$ & & \\
\hline
\end{tabular}

CI: $95 \%$ confidence interval

\section{DISCUSSION}

Methods that use reactions between different dyes and diphenhydramine are based on the formation of a complex between anionic dyes and positively charged basic groups in acid medium, which results in a coloured complex with strong absorption in the visible range of electromagnetic spectrum [8-11]. Although they present appropriate parameters of validation, they also exhibit some limitations in formulations where active ingredients are mixed with diphenhydramine-like basic groups. These active ingredients react in a similar way [16] and therefore hinder their individual determinations by means of this methodology. 
Methods for determination of diphenhydramine syrup by HPLC or capillary electrophoresis that only require syrup dilution are simple and fulfil requirements of validation [3-5]. However, the total absence of sample treatment entails injecting smaller or higher concentrations of a series of components, such as flavouring agents, saccharose or aspartame, acidulates, natural or artificial colours and preservatives, into the chromatography system. The presence of these components in the analytic sample combined with a lower selective detection wavelength $(210-215 \mathrm{~nm})$, could present interference problems during diphenhydramine determination in some formulations.

In this work, the selective separation of basic compounds from of the syrup excipients was achieved. In the sample treatment lidocaine hydrochloride (internal standard) was submitted to extraction with excellent recovery. Additionally, the recovery for dextromethorphan hydrobromide solutions was also evaluated, since this compound is frequently considered to be associated with diphenhydramine and the cartridge was found to have an excellent behaviour with said compound. Once the compounds were recovered and were free of syrup components, an excellent chromatographic separation of the compounds of interest was achieved at a wavelength with a lower possibility of interferences.

The proposed method represents an alternative for analysing diphenhydramine hydrochloride syrup by using an easy and fast treatment (less than $5 \mathrm{~min}$ ) that offers selectivity from the very extraction. The SPE is a feasible and simple alternative for treating samples of diverse origin that saves several resources (time and solvents). The type of cartridge selected in this method was a modified polymeric resin made of polystyrene-polydivinylbenzene, which by means of sulfonation turns into a selective ion-exchange cartridge for basic compounds [13]. Therefore, it allows separating selectively the compound of interest and the internal standard from the other components of the formulation.

\section{CONCLUSION}

It is important to choose simpler methods of analysis, whose performance is more advantageous and less polluting. The appropriate pre-treatment of the samples will always provide a reliable analytic result. Despite a broad variety of methods reported for different analytes, an alternative characterized by simplicity and reliability will always have significant application.

\section{CONFLICT OF INTERESTS}

All authors have none to declare

\section{REFERENCES}

1. Hardman JG, Limbird LE. Goodman y Gilman. Las Bases Farmacológicas de la Terapéutica. 10 ${ }^{\mathrm{a}}$ Ed. Buenos Aires: Mc Graw Hill; 2003. p. 664-6.

2. Gennaro AR. Remington Farmacia. 19a Ed. Buenos Aires; Ed. Médica Panamericana; 1999. p. 1860-1.
3. Gomez MR, Olsina RA, Martínez LD, Silva MF. Simultaneous determination of dextromethorphan, diphenhydramine and phenylephrine in expectorant and decongestant syrups by capillary electrophoresis. J Pharm Biomed Anal 2002;30:791-9.

4. Marchesini AF, Willner MR, Mantovani VE, Robles JC, Goicoechea HC. Simultaneous determinations of naphazoline, diphenhydramine and phenylephrine in nasal solutions by capillary electrophoresis. J Pharm Biomed Anal 2003;31:39-46.

5. Çaglar H, Büyüktuncel E. HPLC method development and validation: simultaneous determination of active ingredients in a cough and cold pharmaceuticals. Int J Pharm Pharm Sci 2014;6:421-8.

6. Moffat AC, Osselton MD, Widdop B. Clarke's analysis of drugs and poisons in pharmaceuticals body fluids and postmortem material 4th Ed. London; Pharmaceutical Press; 2011. p. 1273.

7. Farmacopea de los Estados Unidos Mexicanos. 11 ${ }^{\mathrm{a}}$ Ed. Mexico DF. Secretaría de Salud; 2014. p. 1775-6.

8. El-Didamony AM, Moustafa MA. Spectrophotometric determination of diphenhydramine hydrochloride in pharmaceutical preparations and biological fluids via ion-pair formation. Arab J Chem 2010;3:265-70.

9. Tripparat P, Lapanantnoppakhun S, Jakmunee J, Grudpan K. Determination of diphenhydramine hydrochloride in some single tertiary alkylamine pharmaceutical preparations by flow injection spectrophotometry. J Pharm Biomed Anal 2002;30:105-12.

10. Ulu ST, Elmali FT. Spectrophotometric method for the determination, validation, spectroscopic and thermal analysis of diphenhydramine in a pharmaceutical preparation. Spectrochim Acta Part A 2010;77:324-9.

11. El-Didamony AM, Hafeez SM. Extractive spectrophotometric determination of some antihistaminic drugs from pharmaceutical formulations using rose bengal. Int J Pharm Pharm Sci 2015;7:152-7.

12. Huck CW, Bonn GK. Recent developments in polymers-based sorbents for solid-phase extraction. J Chromatogr A 2000;885:51-72.

13. Leon-Gonzalez ME, Perez-Arribas LV. Chemically modified polymeric sorbents for sample preconcentration. J Chromatogr A 2000;902:3 -16.

14. ICH Harmonised Tripartite Guidelines. Validation of Analytical Procedures: Text and Methodology Q2 (R1). International Conference on Harmonization; 2005. Available from: http://www.ich.org/fileadmin/Public_Web_Site/ICH_Products /Guidelines/Quality/Q2_R1/Step4/Q2_R1_Guideline.pdf. [Last accessed on 05 Jul 2017]

15. Calabuig-Hernandez S, Garcia-Alvarez-Coque MC, Ruiz-Angel MJ. Performance of amine as silanol suppressors in reversed-phase liquid chromatography. J Chromatogr A 2016;1465:98-106.

16. El-Didamony AM, Hafeez SM, Saad AA. Extractionspectrophotometric determination of some antihypertensive drugs in pharmaceutical and biological fluids using two sulphonphthalein dyes. Int J Appl Pharm 2015;7:10-7. 